\title{
X-ray Synchrotron Studies of Nanostructure Formation in High Temperature - Pressure Treated Silicon Implanted with Hydrogen
}

\author{
K. Wieteska ${ }^{a}$, W. Wierzchowski ${ }^{b}$, W. GraefF ${ }^{c}, A \cdot$ Misiuk $^{d}$, \\ A. BARCZ ${ }^{d}$, L. Bryja ${ }^{e}$ AND V.P. POPOV $^{f}$ \\ ${ }^{a}$ Institute of Atomic Energy, 05-400 Otwock-Świerk, Poland \\ ${ }^{b}$ Institute of Electronic Materials Technology \\ Wólczyńska 133, 01-919 Warsaw, Poland \\ ${ }^{c}$ HASYLAB at DESY, Notkestraße 85, 22603 Hamburg, Germany \\ ${ }^{d}$ Institute of Electron Technology, al. Lotników 32/46, 02-668 Warsaw, Poland \\ ${ }^{e}$ Wrocław Technical University, Wyb. Wyspiańskiego 27, 53-370 Wrocław, Poland \\ ${ }^{f}$ Institute of Semiconductor Physics, RAS \\ Lavrentieva 13, 630090 Novosibirsk, Russia
}

\begin{abstract}
The effects of various high temperature-pressure treatments in Czochralski grown silicon $(\mathrm{Cz}-\mathrm{Si})$ implanted with $130 \mathrm{keV}$ hydrogen to the dose of $4 \times 10^{16} \mathrm{~cm}^{-2}$ were investigated using synchrotron X-ray topographic methods and rocking curve measurements. The high temperature-pressure processes included $10 \mathrm{~h}$ annealing at $450^{\circ} \mathrm{C}, 650^{\circ} \mathrm{C}$, and $725^{\circ} \mathrm{C}$ at argon pressure $12 \mathrm{kbar}$ and 1 bar. The topographic investigations were performed with projection and section methods in back-reflection and transmission geometry. It was found that annealing resulted in significantly reduced strain induced by the implantation, which became undetectable with presently used very sensitive synchrotron arrangement. A significant difference between the $\mathrm{Cz}-\mathrm{Si}: \mathrm{H}$ samples annealed at high and atmospheric pressure was observed. In the first case a distinct topographic contrast attributed to the formation of comparatively larger inclusions was observed. This effect was different at different temperatures. The samples annealed at enhanced pressure were more uniform and often produced significant interference effects.
\end{abstract}

PACS numbers: 68.55.-a, 61.46.+w 


\section{Introduction}

The defects induced by hydrogen implantation to silicon can undergo to different transformations in the course of thermal annealing performed at various pressure and temperature conditions. The studies of implantation-induced defects seem to be interesting both in cognitive and practical aspects connected with diffusion and aggregation of hydrogen, leading finally to the formation of nano-dimensional hydrogen inclusions and further splitting of the shot-through layer from the substrate. The last effect is widely used in so-called "smart-cut" technology.

Enhanced hydrostatic pressure (HP) up to $1.2 \mathrm{GPa}$ of argon ambient at annealing (high temperature (HT), at up to $1000 \mathrm{~K}$ ) of $130 \mathrm{keV}$ hydrogen $\mathrm{H}_{2}^{+}$ implanted silicon to the doses up to $4 \times 10^{16} \mathrm{~cm}^{-2}$ resulted in suppression of hydrogen out-diffusion and in stress stimulated creation of small defects. Some of these defects may act as thermal donors. This treatment also resulted in photoluminescence (PL) at infrared, peaking at about $0.8 \mathrm{eV}$ [1]. The transmission electron microscopy (TEM) indicated that the HT-HP treatment of Si:H caused a creation of dense array of clusters and voids in the area corresponding to the hydrogen ion projected range [2], analogous to the defects detected in nanostructural porous silicon. Silicon implanted with $24 \mathrm{keV}$ hydrogen and HP-treated at $870 \mathrm{~K}$ exhibited visible photoluminescence at about $570 \mathrm{~nm}$ with intensity increasing with HP [3], similarly as detected in nanostructural porous silicon.

In the present work differently HP-treated hydrogen implanted silicon samples are studied with a number of synchrotron topographic methods, especially the Bragg-case section topography. The samples were additionally studied with transmission electron microscopy (TEM), secondary ion mass spectroscopy (SIMS) and photoluminescence measurements.

\section{Experimental}

$\mathrm{Cz}-\mathrm{Si}$ wafers of (001) orientation, with the concentration of interstitial oxygen, $c_{0}$, up to $1 \times 10^{18} \mathrm{~cm}^{-3}$, were implanted with hydrogen $\left(\mathrm{Cz}-\mathrm{Si}: \mathrm{H}_{2}^{+}\right.$dose $4 \times 10^{16} \mathrm{~cm}^{-2}$, energy $130 \mathrm{keV}$, projected range $\left.R_{\mathrm{p}}=0.65 \mu \mathrm{m}\right)$. The implanted wafers were treated under Ar pressure up to $1.2 \mathrm{GPa}$ at temperatures up to $1000 \mathrm{~K}$ for up to $10 \mathrm{~h}$.

The most important method of characterization was synchrotron white beam Bragg-case section topography realized at HASYLAB. The samples were also studied by rocking curve measurements using a $1.1 \AA$ mononochromatized synchrotron radiation with a small $50 \mu \mathrm{m}$ probe beam diameter. The additional studies of the defect structure were performed with TEM while the hydrogen distribution was studied with the SIMS method. The additional photoluminescence measurements in the infrared range were also performed. 


\section{Results and discussion}

As is illustrated by the representative rocking curves shown in Fig. 1 the unnegligeable implantation-induced strain was observed only in the as-implanted state. As may be evaluated from the rocking curves, the annealing performed at temperatures of $870 \mathrm{~K}$ and higher reduced the strain value below $0.5 \times 10^{-6}$.
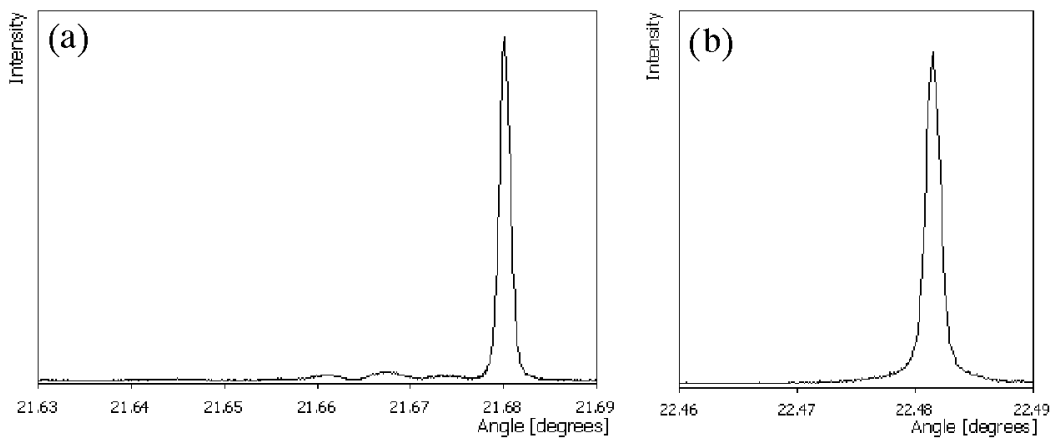

Fig. 1. The representative synchrotron rocking curves in 400 reflection of $1.1 \AA$ radiation of silicon samples implanted with $130 \mathrm{keV}$ hydrogen to the dose of $4 \times 10^{16} \mathrm{~cm}^{-2}$ : (a) as-implanted, (b) after $1 \mathrm{~h}$ annealing under $10^{5} \mathrm{~Pa}$ at $870 \mathrm{~K}$.

The Bragg-case section investigations enabled very effective monitoring of the formation of hydrogen inclusions corresponding to local exfoliation of the implanted layer. Usually the samples free of exfoliation produced distinct "Chukhovski-Zaumseil" interference fringes characteristic of Bragg-case section patterns in elastically bent crystals. As may be followed in the topographs reported in Figs. 2a-e, these fringes vanished or were disturbed after HP-annealing processes. The dampening of the fringes took place after relatively short annealing performed at atmospheric pressure. With HP this decomposition required respectively a longer annealing time. Irregularities of fringes on some topographs are caused by non-regular bending caused by accidental defects on the edges of the samples.

Similarly as in the case [4] the strain profile in the as-implanted Si:H sample seems to be caused mainly by the point defect (interstitial) distribution, which is, however, similar to the distribution of hydrogen. In the present case the distribution of hydrogen was controlled directly with SIMS measurements. The representative distributions of hydrogen in HT-HP treated samples are shown in Fig. 3. It can be noticed that the present implantation contain a small additional component of $\mathrm{H}^{+}$ions with a significantly higher penetration range. After thermal annealing we may observe a tendency of hydrogen gathering at mostly deformed region, which is most distinct at lower pressure. This phenomenon as well as a lower level of exfoliation are well explainable by lower diffusion of hydrogen under enhanced pressure. 
(a)

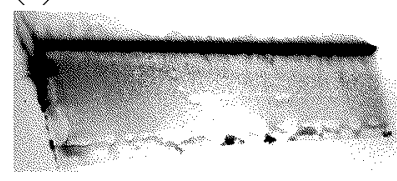

(d) (b)

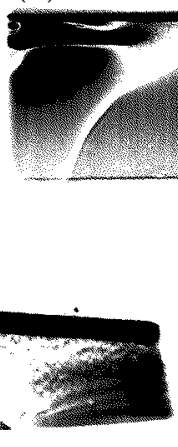

(c)

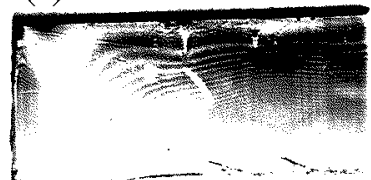

(e)

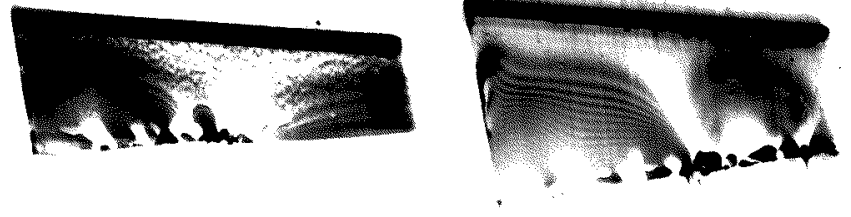

Fig. 2. Synchrotron white beam Bragg-case section topographs of silicon samples implanted with $130 \mathrm{keV}$ hydrogen to the dose of $4 \times 10^{16} \mathrm{~cm}^{-2}$ after HP-HT treatment: (a) $920 \mathrm{~K}, 10^{5} \mathrm{~Pa} 0.5 \mathrm{~h}$; (b) $920 \mathrm{~K}, 1.2 \mathrm{GPa}, 0.5 \mathrm{~h}$; (c) $920 \mathrm{~K}, 1.2 \mathrm{GPa}, 10 \mathrm{~h}$; (d) $1000 \mathrm{~K}$, $10^{5} \mathrm{~Pa}, 10 \mathrm{~h}$; (e) $1000 \mathrm{~K}, 1.1 \mathrm{GPa}, 10 \mathrm{~h}$.
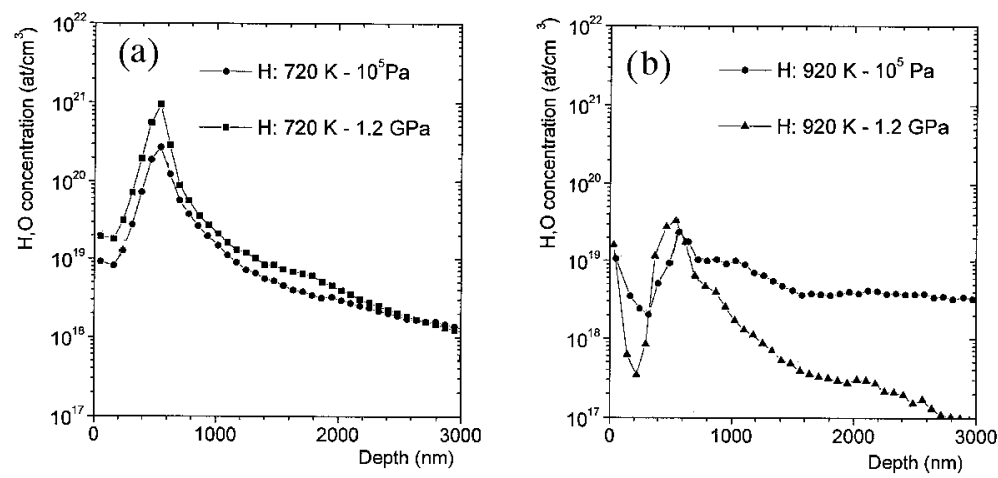

Fig. 3. The hydrogen distribution in the HP-HT treated samples obtained with the SIMS method: (a) for $720 \mathrm{~K}$, (b) for $920 \mathrm{~K}$.

The representative TEM picture of the Si:H sample is shown in Fig. 4. The sample was annealed for one hour at $10^{5} \mathrm{~Pa}$ at $920 \mathrm{~K}$. The picture of the cross-section of the sample exhibits a large concentration of defects with distinct small tracks in the mostly deformed region situated at about $0.55 \mu \mathrm{m}$ below the surface. Below this region we see a wide spread distribution of less dense separated defects with a maximal concentration at approximately $1.1 \mu \mathrm{m}$ below the surface, corresponding to maximum of the distribution of $\mathrm{H}^{+}$ions ranges.

The photoluminescence spectra of presently HT-HP treated samples are shown in Fig. 5. The origin of PL at $0.8 \mathrm{eV}$ is not known; that at 0.94 can be attributed to vacancy clusters in a form of small bubbles filled with hydrogen [5] 


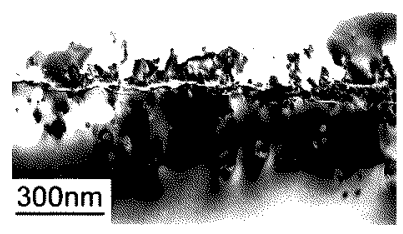

Fig. 4. The TEM picture of the sample annealed $10 \mathrm{~h}$ at $920 \mathrm{~K}$ at atmospheric pressure.

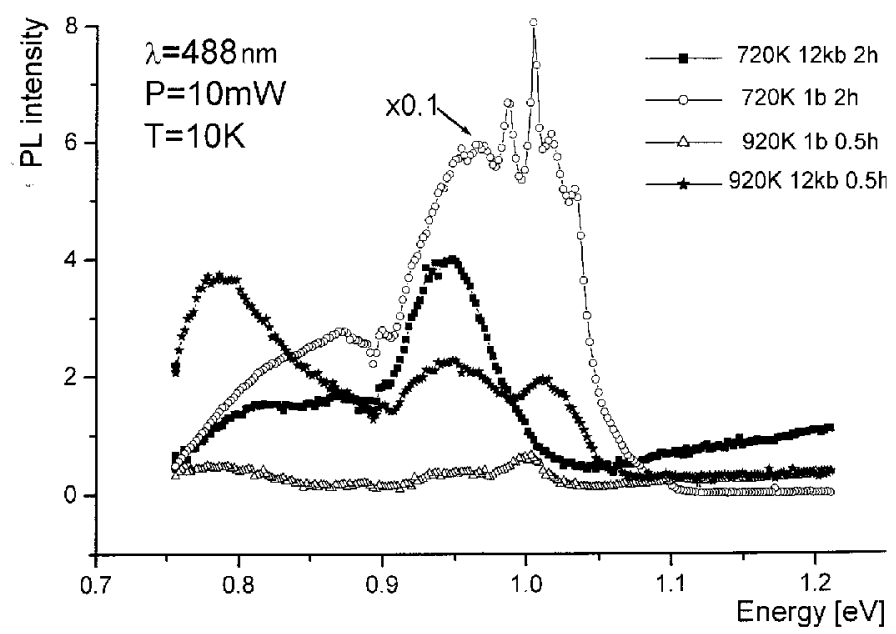

Fig. 5. The representative photoluminescence spectra for samples annealed at 720 and $920 \mathrm{~K}$.

or to dislocations-related D3 line. The broad peak at $1.0 \mathrm{eV}$ for the sample annealed at $720 \mathrm{~K}$ can also be attributed to dislocations-related photoluminescence (D4 line). Its width may indicate the considerable non-uniform strain in the lattice.

\section{Conclusions}

The silicon implanted with $130 \mathrm{keV}$ hydrogen to the dose of $4 \times 10^{16} \mathrm{~cm}^{-2}$ and variously HP treated was studied with a number of characterization methods including synchrotron X-ray diffraction methods. The use of white beam synchrotron section topography enabled the examination of the formation of hydrogen precipitate and local exfoliation.

It was found that enhanced pressure at annealing significantly reduced the velocity of the formation of hydrogen precipitates and exfoliation process. The samples annealed under a higher pressure exhibited the additional PL maximum at $1.0 \mathrm{eV}$ similar to those observed in silicon with a large concentration of dislocation.

The SIMS measurement indicated a smaller decrease in concentration of hydrogen for the samples annealed at higher pressures. 


\section{Acknowledgments}

The present work was partly supported by the State Committee for Scientific Research. The authors are thankful to Prof. A. Romano-Rodriguez from Barcelona University for TEM results.

\section{References}

[1] A. Misiuk, H.B. Surma, I.V. Antonova, V.P. Popov, J. Bak-Misiuk, M. Lopez, A. Romano-Rodriguez, A. Barcz, J. Jun, Solid State Phen. 69-70, 345 (1999).

[2] A. Misiuk, J. Bak-Misiuk, A. Barcz, A. Romano-Rodriguez, I.V. Antonova, V.P. Popov, C.A. Londos, J. Jun, Int. J. Hydrog. Energy 26, 483 (2001).

[3] K.S. Zhuravlev, I.E. Tyschenko, private communication.

[4] K. Wieteska, W. Wierzchowski, W. Graeff, K. Dłużewska, Phys. Status Solidi A 168, 11 (1998).

[5] V. Rainieri, S. Coffa, E. Szilagyi, J. Gyulai, E. Rimini, Phys. Rev. B 61, 937 $(2000)$. 